\title{
Erratum to: \\ The Ageing of Materials and Structures
}

Klaas van Breugel, Dessi Koleva and Ton van Beek

\section{Erratum to:}

K. van Breugel et al. (eds.), The Ageing of Materials

and Structures, https://doi.org/10.1007/978-3-319-70194-3

In the original version of the book, the incorrectly published author name "Ton Beek" has been now corrected to read as "Ton van Beek" in Cover, Copyright and Title pages. 